\title{
Image Labeling and Segmentation using Hierarchical Conditional Random Field Model
}

\author{
Mr. Manoj K. Vairalkar ${ }^{1}$ and Mrs.Sonali.Nimbhorkar ${ }^{2}$ \\ ${ }^{1}$ Department of Computer Science and Engineering, RTMN University,Nagpur \\ mkvairalkar@gmail.com \\ 2 Department of Computer Science and Engineering, RTMN University,Nagpur \\ nimsonali21@yahoo.com
}

\begin{abstract}
The use of hierarchical Conditional Random Field model deal with the problem of labeling images. At the time of labeling a new image, selection of the nearest cluster and using the related CRF model to label this image. When one give input image, one first use the CRF model to get initial pixel labels then finding the cluster with most similar images. Then at last relabeling the input image by the CRF model associated with this cluster. This paper presents a approach to label and segment specific image having correct information.
\end{abstract}

\section{KEYWORDS}

CRF, Label Descriptor, wavelet transform

\section{Introduction}

Segmentation is the decomposition of an image into these objects and regions by associating every pixel with the object that it corresponds to. Most humans can easily segment an image. Computer automated segmentation is a difficult problem, requiring sophisticated algorithms that work in tandem.

labeling identifies an object record of an information page based on the labeling of object elements within an object record and labels object elements based on the identification of an object record that contains the object elements.

The understanding of image and object identification is the core technology. Here the goal is to assign every pixel of the image with an object class label. For this the solutions fall into two general categories: parametric methods and nonparametric methods.

Parametric methods [1] mostly rivet minimizing a Conditional Random Field (CRF) model to assign a particular label to every pixel on the basis of probability. In learning phase of parametric method, the parameters of the CRF models are optimized with the help of training examples, and in an inference phase, the CRF model is useful to label a test image. In difference to parametric methods, nonparametric methods [10] do not involve any training. The design of these methods is to relocate labels from a recovery set which contains meaningful similar images. Here, the approach is to label specific type of image whose correct information is present.

DOI : 10.5121/ijcsit.2011.3616 


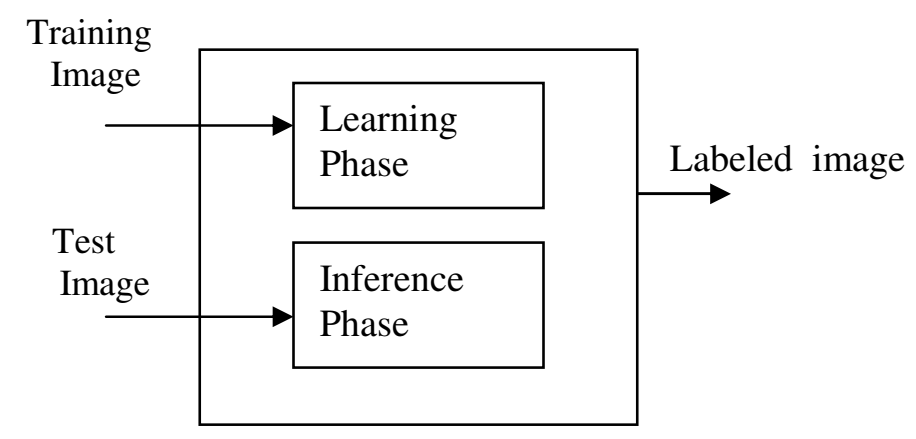

Fig.1: Conditional Random Field Model

In learning phase, firstly the training of image to the system carried out then according to the test image type, the inference phase tries to matches input image with trained system on the basis of probability.

\section{Related Work}

\subsection{Parametric methods}

Image labeling by optimizing a CRF model has proven to be the state-of-the-art parametric image labeling method. Traditional CRF models combine unary energy terms, which evaluate the possibility of a single pixel taking a particular label, and pair-wise energy terms, which evaluate the probability of adjacent pixels taking different labels. Although these approaches work well in many cases, they still have their own limitations because these CRF models are only up to second-order and it is difficult to incorporate large-scale contextual information.

\subsection{Nonparametric methods}

The key components of nonparametric methods are how to find the retrieval set which contains similar images, and how to build pixel-wise or super pixel-wise links between the input image and images in the retrieval set. 
International Journal of Computer Science \& Information Technology (IJCSIT) Vol 3, No 6, Dec 2011

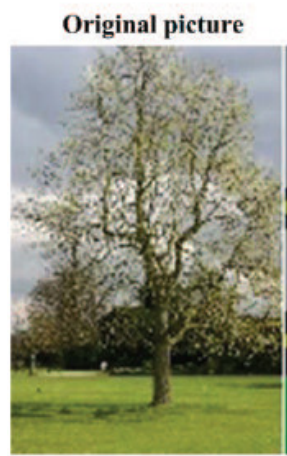

(a)

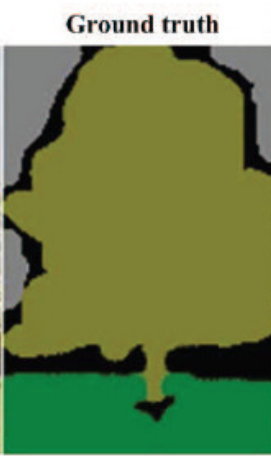

(b)

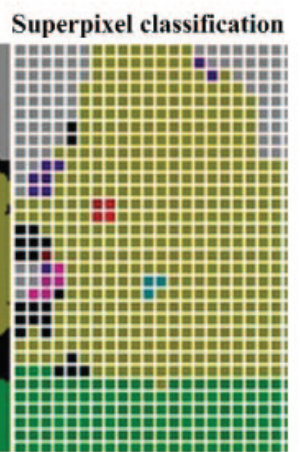

(c)

Fig.2: Illustration of super pixel classification

\section{Modeling Methods}

\subsection{Label Based Descriptor}

By labeled image, each pixel is labeled as one of the $k$ object classes $L=\left\{m_{k}\right\}$. The meaningful data of an image is obtained from the position, appearance and shape of each object in the image. Here, the value of can be varied.

\subsection{Positional information}

The positional information can be obtained by dividing image, grid. When we divide image it forms cell formulation. Within each grid cell say gij, calculate the distribution Sijk of every object class $\mathrm{m}_{k} \in L$. Then assemble all the cell coverage information into a vector $\mathrm{d} p I$ of length $K(n p)^{2}$. A big $n p$ would make $\mathrm{d}^{p} I$ capture the positional information more precisely, while a small $n p$ would make d $p$ I less sensitive to image displacement and classification errors. If $\mathrm{L}$ contains 3 objects and grid value is 3 then the calculation yields to 27.If L contains 3 objects then the representation is

$\mathrm{L}=\left\{\mathrm{m}_{0}, \mathrm{~m}_{1}, \mathrm{~m}_{2}\right\}$

Where $\mathrm{m}_{0}$ can be treated as object $1, \mathrm{~m}_{1}$ can be represented as object 2 and so on The partitioning of $9 \times 9$ image represented as:

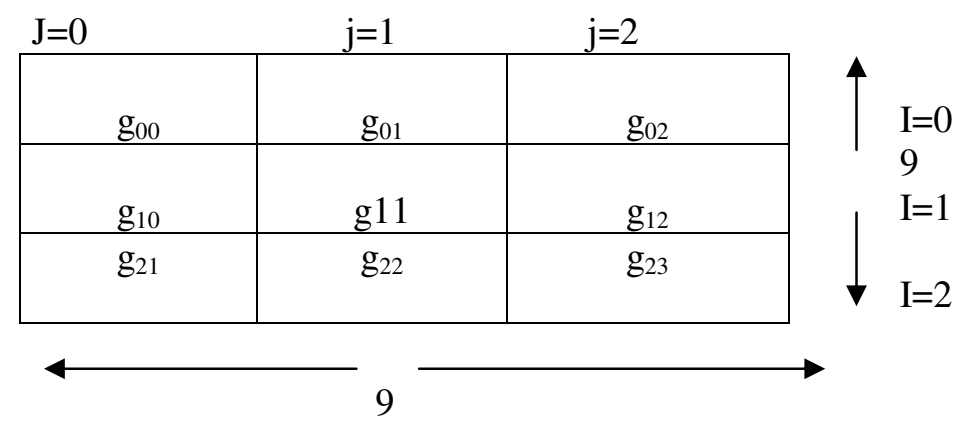




\subsection{Appearance}

The appearance information obtained by calculating the mean color cijk $=(r i j k$, gijk, bijk $)$ of each object class $c k$ within each cell gij. To stabilize the mean color statistics, scale each mean color cijk as pijk cijk. Again, all mean colors cijk are collected into a vector dcI of length $3 K(n p) 2$.

\section{Image Loading}

In image loading module, the Java Advanced Imaging Application Program Interface is used that allows reading and writing of various image formats, and helps for manipulation. Then getting the Red, Green and Blue value for given image one is able to binaries the image.

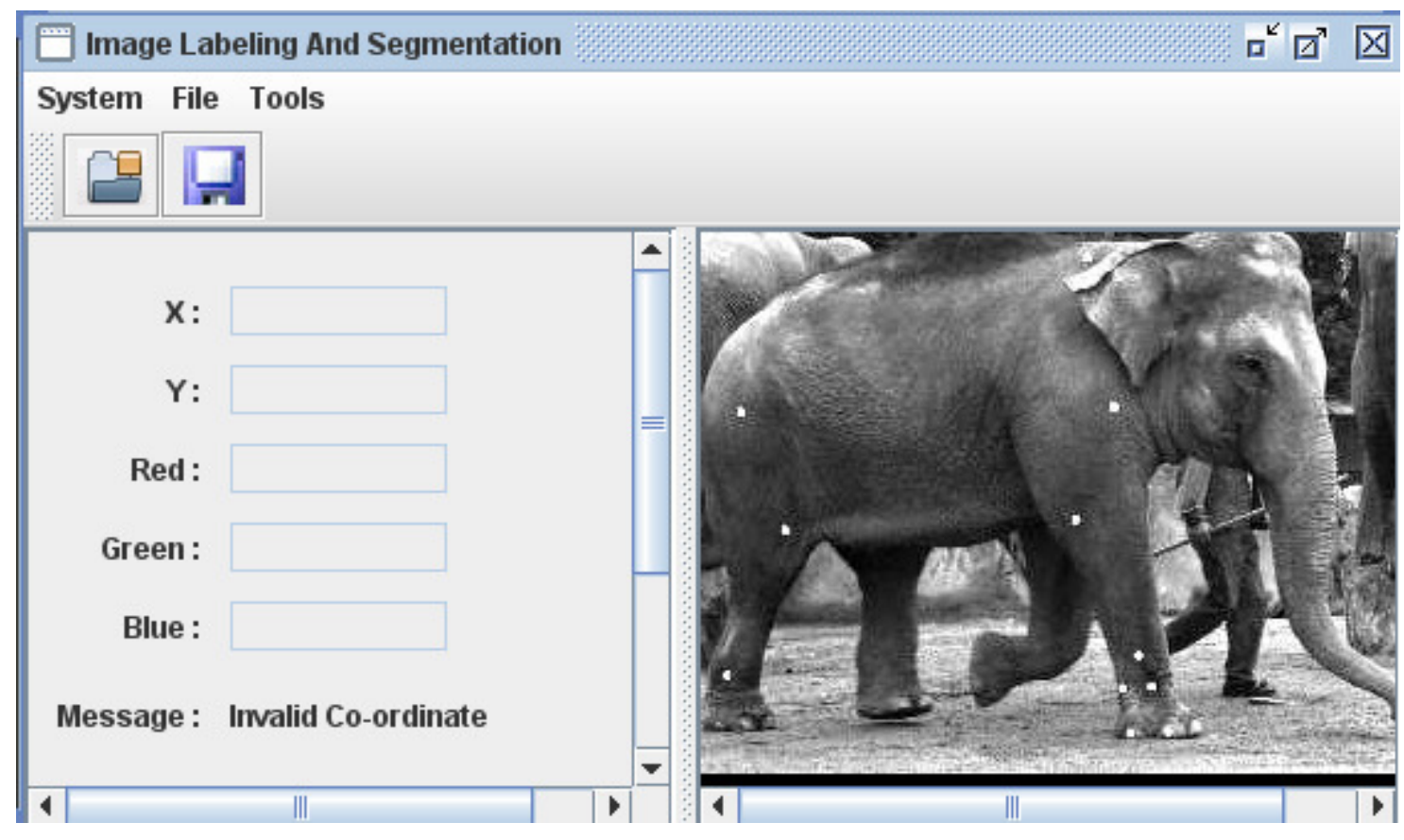

Fig.3: Image Loading Sample 


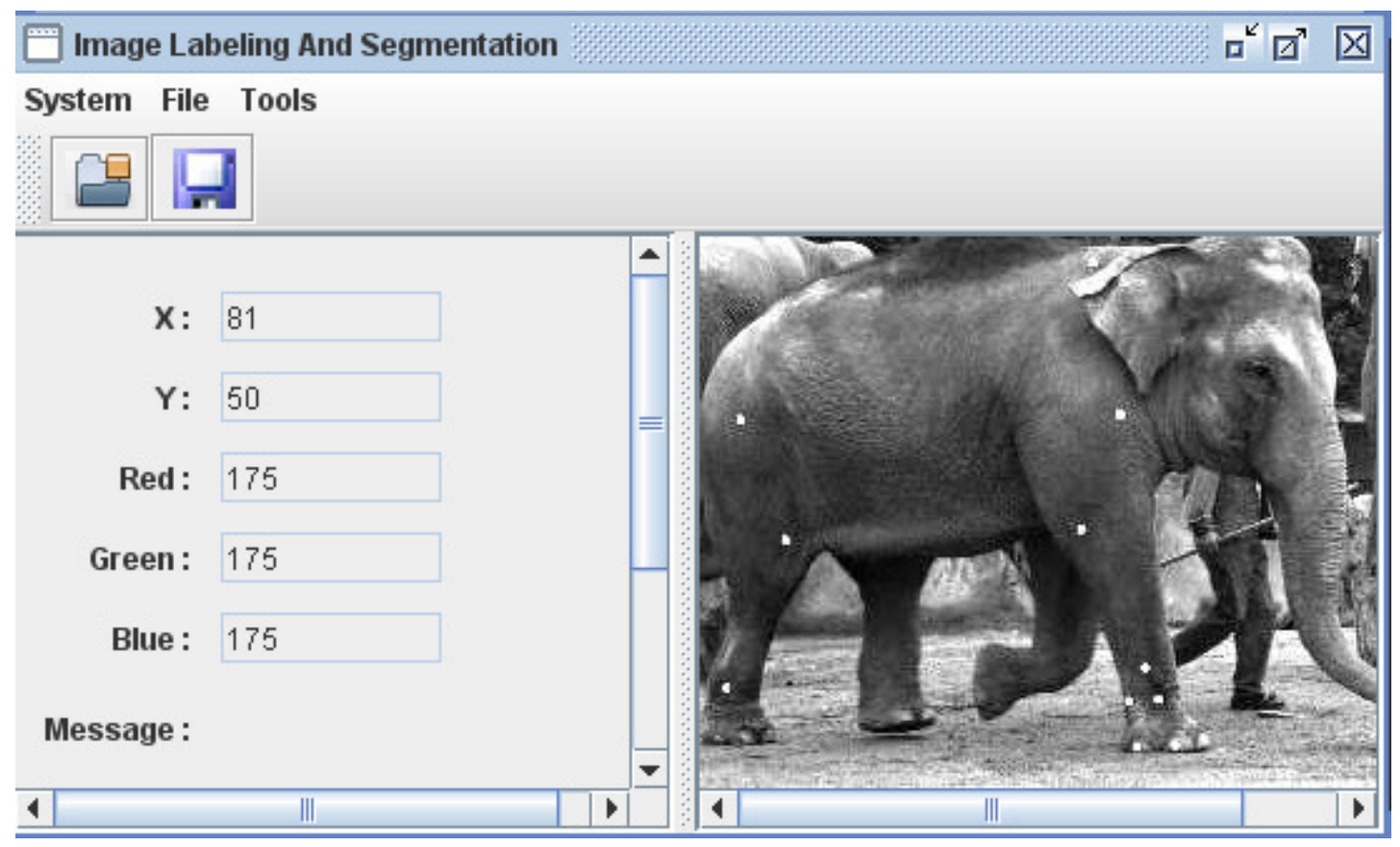

Fig.4: Image Binarization

The flow of process of image loading and binarization shown as follows. 


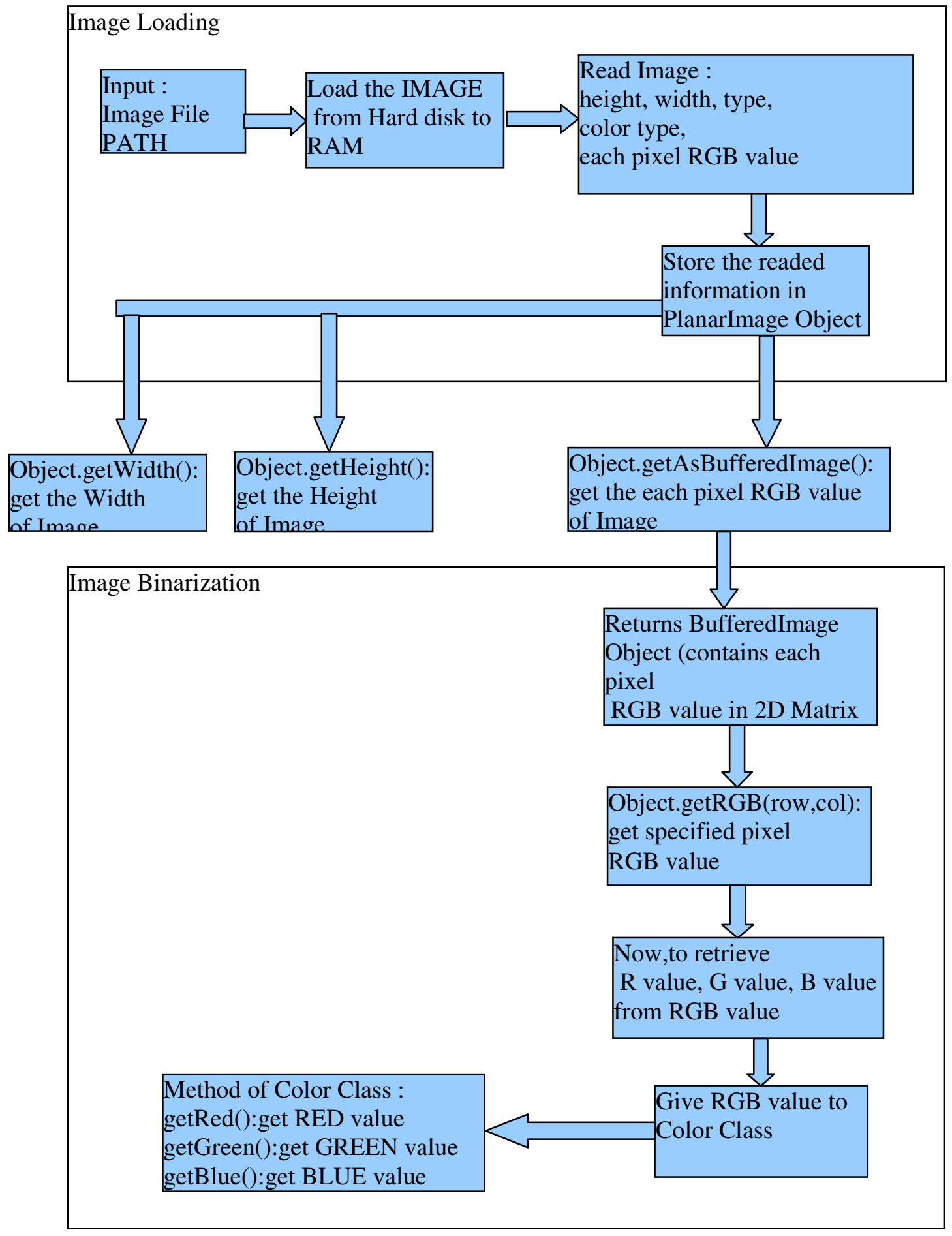

Fig.5: Block Diagram for Image Loading and Binarization 


\section{Segmentation}

Segmentation refers to the practice of partition a digital image into various segments. The objective of segmentation is to simplify and/or modify the depiction of an image into something that is more significant and easier to analyze. Image segmentation is used to locate objects and boundaries (lines, curves, etc.) in images. More precisely, image segmentation is the process of assigning a label to every pixel in an image such that pixels with the same label share certain image characteristics.

The result of image segmentation is a set of segments that collectively cover the entire image, or a set of contours extracted from the image. Each of the pixels in a area are like with respect to some characteristic such as color, intensity, or texture. Color image segmentation is useful in many applications. From the segmentation results, it is possible to identify regions of interest and objects in the scene, which is very beneficial to the successive image analysis.
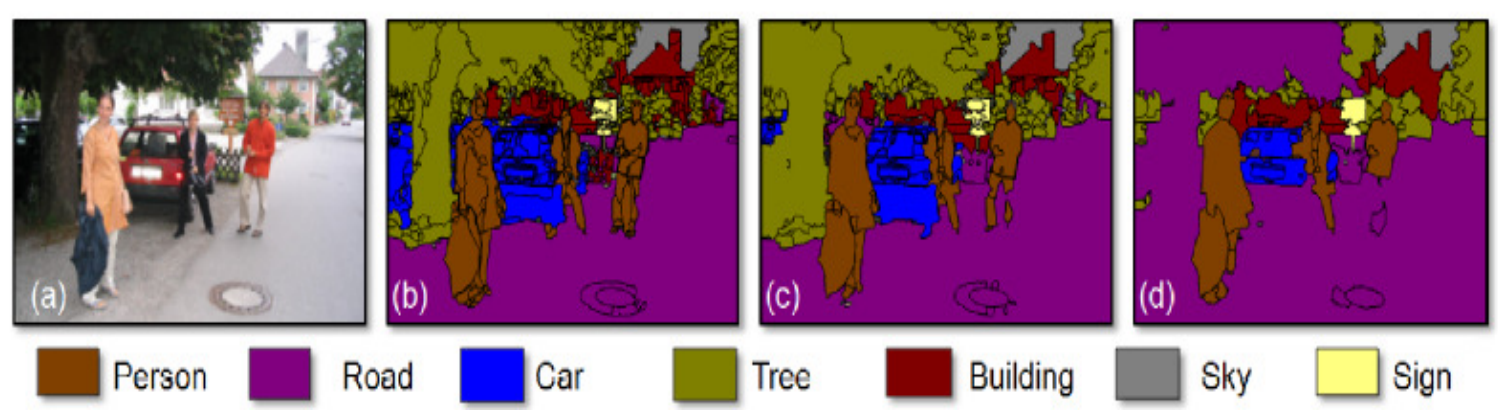

Fig.6.Image Labeling and segmentation

\section{Wavelet Transform}

The optimization of speed performance can be achieved with the help of wavelet transform as compression is possible. Wavelets are mathematical functions that scratch up data into unlike frequency components, and then study each component with a resolution matched to its scale. There are advantages over traditional Fourier methods in analysing physical situations where the signal contains discontinuities and sharp spikes. Wavelets were developed independently in the fields of mathematics, quantum physics, and electrical engineering. Wavelets are functions that satisfy certain mathematical requirements and are used in representing data or other functions. This idea is not new. Approximation using superposition of functions The wavelet transform is a mathematical tool that decomposes a signal into a representation that shows signal details and trend as a function of time. One use this representation to characterize temporary events, decrease noise, compress data, and perform many other operations. The main advantage of wavelet methods over traditional Fourier methods are the use of localized basis functions and the faster computation speed. Localized basis functions are ideal for analysing real material situations in which a signal contains discontinuities and sharp spikes. Wavelet transform is an evolving technology which offers far higher degrees of data compression compared to standard transforms 
International Journal of Computer Science \& Information Technology (IJCSIT) Vol 3, No 6, Dec 2011

\section{Conclusion}

In this paper, presents an approach to label the images whose correct information is present. The Hierarchical CRF model finds similar object from trained examples and assign suitable priority according to object to label. The experimental results looks as figure (1) naming Effect of image quantisation on object segmentation where (a) Original image. (b)-(d) Object class segmentations with different images. (b), (c) and (d) use three different unverified segmentations of the image. Each segment is assigned the label of the dominant object present in it. It can be seen that (b) is the best for tree, road, and car. However, (d) is better for the left person and the sign board.

\section{References}

[1]C. Galleguillos, A. Rabinovich,S. Belongie "Object categorization using co-occurrence, location and appearance in Computer Vision and Pattern Recognition", IEEE 2008

[2] Thomas Deselaers, vittorio,"Visual and semantic similarity in ImageNet", IEEE 2010

[3] Gemma Roig,Xavier Boix,Joan serrat," Hierarchical CRF with Product Label Spaces for Parts-based Models", IEEE 2010

[4] Jun Tang,” A color Image Segmentation Based on Region Growing”, IEEE 2010

[5] Hichem Sahbi,"Superpixel-Based Object Class Segmentation Using Conditional Random Fields", ICASSP IEEE 2011

[6] Yushi Jing , Michele Covell,"Comparision Of Clustering Approaches For Summarizing Large Populations Of Images", IEEE 2010

[7] Jordan Reynolds ,Kevin Murphy," Figure-ground segmentation using a hierarchical conditional random field", IEEE 2007

[8] Lubor Ladickly ,Chris Russell,”Associative Hierarchical CRF for Object Class Image Segmentation”, IEEE 12th International Conference on Computer Vision 2009

[9] Qixing Huang, Mei Han, Bo Wu,Sergey Ioffe," A Hierarchical Conditional Random Field Model for Labeling and Segmenting Images of Street Scenes",2011

[10] Ce Liu , Jenny Yuen,”Nonparametric scene Parsing:Label Transfer via Dense Scene Alignment”, IEEE 2009

[11] Peng Lu , He Ren,"Hierarchical Conditional Random Fields For Chinese Named Entity Tagging”, IEEE 2009

[12] William Freeman , Mark Rubin," Context-based vision system for place and object recognition", ICCV'03, IEEE 2003

[13] B. C. Russell, A. Torralba, K. P. Murphy, W. T. Freeman.LabelMe: A Database and Web-Based Tool for Image Annotation. International Journal of Computer Vision.

[14] Lawrence Y. Deng, Jason C. Hung "Real-time Hand Gesture Recognition by Shape Context Based Matching and Cost Matrix" IEEE 2011

[15] Chen Zhou, Zhengguang Xu, Benxiong Huang,"Activity Recognition from Call Detail Record:

Relation Between Mobile Behavior Pattern And Social Attribute Using Hierarchical Conditional

Random Fields",IEEE 2010

[16] Meng Wang, Janusz Konrad, Prakash Ishwar, Kevin Jing, Henry Rowley,'Image Saliency: From Intrinsic to Extrinsic Context”, IEEE2010

[17] Gustavo Carneiro, Antoni B. Chan, Pedro J. Moreno, Nuno Vasconcelos,," Supervised Learning of Semantic Classes for Image Annotation and Retrieval", IEEE 2007

[18] Luo Ronghua, Min Huaqing," Hybrid Conditional Random Fields for Multi-object Tracking with a Mobile Robot", IEEE 2010

[19] Luis von Ahn and Laura Dabbish," Labeling Images with a Computer Game”, IEEE 2004

[20] Paul Schnitzspan, Mario Fritz, Stefan Roth, Bernt Schiele," Discriminative Structure Learning of Hierarchical Representations for Object Detection”,IEEE 2008 